\title{
Physical Exercise Combined With CPAP in Subjects Who Underwent Surgical Myocardial Revascularization: A Randomized Clinical Trial
}

\author{
Pollyana Windmöller, Emely Teixeira Bodnar, Julia Casagrande, Fernanda Dallazen, \\ Juliana Schneider, Silvana Agnolleto Berwanger, Audrey Borghi-Silva, and \\ Eliane Roseli Winkelmann
}

\begin{abstract}
BACKGROUND: Aerobic exercise and CPAP benefit patients in the postoperative period of cardiac surgery. To our knowledge, the association of aerobic exercise on an exercise bicycle with CPAP has not yet been demonstrated. Therefore, we aimed to evaluate the effectiveness of physical exercise on a cycle ergometer combined with CPAP in the postoperative period after coronary artery bypass graft surgery. METHODS: This was a randomized clinical trial, with recruitment from May 2017 to December 2017 (registered in the Brazilian Clinical Trials Registry: RBR69CDYF). The step group ( $n=16$ subjects) started rehabilitation in the immediate postoperative period with breathing exercises and passive mobilization in the sitting position, progressing to active exercises, ambulation, and stair training. For the intervention group ( $n=15$ subjects), dynamic exercises on a cycle ergometer combined with CPAP were added to the step program from the second to the fourth postoperative day in a single daily session. RESULTS: Functional capacity decreased in both groups, but this reduction was not significant in the intervention group $(P=.11)$. The length of stay in ICU was lower in the intervention group $(P=\mathbf{. 0 5 0})$. In both groups there was a decrease in maximum inspiratory and expiratory pressure, as well as in the 1-min sit-to-stand test on the fourth postoperative day compared to the preoperative period. CONCLUSIONS: Physical exercise combined with CPAP promoted the maintenance of functional capacity and reduced the length of stay in the ICU. Key words: physiotherapy; continuous positive airway pressure; aerobic exercise; coronary artery bypass graft surgery; rehabilitation. [Respir Care 2020;65(2):150-157. (C) 2020 Daedalus Enterprises]
\end{abstract}

\section{Introduction}

The number of coronary artery bypass graft surgery $(\mathrm{CABG})$ procedures performed in Brazil is increasing an-

\footnotetext{
Ms Windmöller, Ms Bodnar, and Ms Casagrande, as well as Dr Winkelmann and Dr Dallazen are affiliated with the Undergraduate Program in Physical Therapy at the Regional University of Northwestern Rio Grande do Sul (UNIJUÍ), Ijuí, Brazil. Dr Dallazen, Ms Schneider, and Ms Berwanger are affiliated with the Cardiovascular Surgery Unit of the Heart Institute of Ijuí Charity Hospital, Ijuí, Brazil. Dr Borghi-Silva is affiliated with the Graduate Program in Physical Therapy of the Federal University of São Carlos (UFSCar), São Paulo-SP, Brazil. Ms Windmöller and Dr Winkelmann are also affiliated with the Graduate Program in Comprehensive Health Care (UNICRUZ/UNIJUI), Ijuí, Brazil.
}

A version of this article was presented at the 2018 Physical Therapy Symposium of the Society of Cardiology Congress of Rio Grande do Sul, held August 16-18, 2018, in Gramado, Rio Grande do Sul, Brazil. nually. In 1995, 13,198 surgeries were performed, and in 2015 this number increased $\sim 71 \%$ to 22,559 surgeries. Hospital mortality decreased from $7.6 \%$ to $5.9 \% .{ }^{1}$ This could be explained by greater control of techniques used in surgical procedures, progress in postintervention monitoring, and improvement in hospital care available to patients. ${ }^{2-4}$

Advances in CABG procedures have promoted improvements in patient recovery. However, complications such as

\footnotetext{
The authors have disclosed no conflicts of interest.

Correspondence: Eliane Roseli Winkelmann PhD, Regional University of Northwestern Rio Grande do Sul (UNIJUÍ), Rua do Comércio 3000, Bairro Universitário, 98700-000, Ijuí, Rio Grande do Sul, Brazil. E-mail: elianew@unijui.edu.br.
}

DOI: $10.4187 /$ respcare.06919 
atrial fibrillation, prolonged mechanical ventilation, and the need for re-intervention are factors that negatively affect patient outcomes. ${ }^{5}$ Cardiac rehabilitation seeks to prevent loss of physical capacity and avoid respiratory complications and prolonged bed rest. ${ }^{6}$

During the postoperative period, CPAP has been used to minimize or prevent respiratory complications resulting from the $\mathrm{CABG}$ procedure. One study carried out a physical therapy program included walking and CPAP pressures were set between 10 to $12 \mathrm{~cm} \mathrm{H}_{2} \mathrm{O}$. The main findings of this study showed that the use of CPAP combined with physical training can positively influence exercise tolerance, ventilatory function, and breathing pattern in response to a single bout of exercise after CABG. ${ }^{7}$

The improvement of functional capacity through cardiac rehabilitation seeks to help patients recover and restore their preoperative conditions. ${ }^{8,9}$ The use of physical exercise in the postoperative period of cardiac surgery has demonstrated recovery and maintenance of functional capacity in patients who performed exercises on a cycle ergometer during their hospital stay. ${ }^{10,11}$ In addition to the already widely recognized benefits of postoperative CPAP (eg, improvement of oxygenation, decreased need for endotracheal intubation, and reduction of pulmonary complications ${ }^{12}$ ). However, to our knowledge, cycle ergometer exercise combined with CPAP in the postoperative period of cardiac surgery has not yet been studied. Cycling exercises, with monitoring of cardiac hemodynamics and electrical stability, would allow earlier exercise and could be a safer strategy at this early stage of rehabilitation.

In this context, we aimed to evaluate the effectiveness of physical exercise on a cycle ergometer combined with CPAP in the postoperative period after CABG. Our main hypothesis is that physical exercise on a cycle ergometer combined with CPAP can reduce the stay in the ICU and in the hospital, possibly due to the improvement of functional capacity, respiratory muscle strength, and peripheral muscle resistance.

\section{Methods}

\section{Study Design}

This was a randomized clinical trial conducted in a coronary unit of a hospital in the state of Rio Grande do Sul, Brazil, from May 2017 to December 2017. All subjects were informed of the purpose and the procedures of the study and signed consent prior to participation. The protocol was designed in accordance with the Guidelines and Norms Regulating Research Involving Human Beings according to the National Health Council Resolution 466/12. The protocol was approved by the local research ethics

\section{QUICK LOOK}

\section{Current knowledge}

Protocols using a cycle ergometer and noninvasive ventilation have been studied during the hospital phase of cardiac rehabilitation. Cycling exercises, with monitoring of cardiac hemodynamics and electrical stability, would allow earlier exercise and could be a safer strategy at this early stage of rehabilitation.

\section{What this paper contributes to our knowledge}

This protocol proved to be safe in that no intercurrences were observed during its execution. This protocol is a viable alternative for cardiac rehabilitation in the hospital phase, especially in patients who are incapable of stair training and ambulation. This is noteworthy because the utilization of noninvasive ventilation in association with exercise is poorly explored in these patients.

committee under the CAAE (63135716.3.0000.5350) and registered in the Brazilian Clinical Trials Registry (RBR69CDYF).

\section{Subjects}

Subjects included individuals of both sexes, 40-75 y old (the most frequent age group). Subjects were recruited in the preoperative period and were included in the study if they underwent myocardial revascularization surgery, received evaluation by the physical therapist, received medical consent, and agreed to participate in the study. Subjects were excluded if they were unable to understand and follow the research procedures (ie, the pre- and postevaluation protocol and the research intervention protocol) or they had complications (eg, atrial fibrillation, prolonged mechanical ventilation, or death) in the postoperative period.

\section{Sample Size and Randomization}

Based on the results of a previous study ${ }^{11}$ and assuming that subjects following the step program had a stable 6-min walk test (6MWT), we estimated a sample size of 15 subjects per group to achieve a statistical power of $80 \%$ to detect a $10 \%$ difference in the distance walked. Subjects were randomly assigned by simple randomization (ie, random assignments by raffle with sealed envelopes) in a ratio of $1: 1$ to receive treatment in the step group or the intervention group. Neither the subjects nor the physical 
therapists who supervised the sessions were blinded. However, the investigators who performed the measurements were blinded to treatment allocation.

\section{Outcomes}

The primary outcome of this study was the distance walked in the 6MWT. The secondary outcomes were respiratory muscle strength, lower limbs muscle resistance, mechanical ventilation time, length of stay in the ICU, and length of hospital stay.

\section{Clinical Evaluation of the Sample}

In the preoperative period (ie, on the day before surgery), a direct interview was conducted with each subject. Data collected included sex, age, ejection fraction, presence of cardiovascular risk factors, and comorbidities such as systemic arterial hypertension, diabetes mellitus, dyslipidemia, and COPD. For the evaluation of anthropometric measurements, weight $(\mathrm{kg})$, height $(\mathrm{m})$, and body mass index $\left(\mathrm{kg} / \mathrm{m}^{2}\right)$ were measured. ${ }^{13}$

The postoperative data regarding the surgical time, mechanical ventilation time, number of bypasses in the cardiac surgery, ICU length of stay, and hospital length of stay were collected from the each subject's medical records.

Primary and secondary outcomes were assessed in the preoperative (ie, the day before surgery) and postoperative (postoperative day 4) periods.

\section{6-Min Walk Test}

The submaximal functional capacity was evaluated with the $6 \mathrm{MWT},{ }^{14}$ in which the greatest distance the subject was able to walk in a fixed time interval of 6 min was measured along a hallway $30 \mathrm{~m}$ in length. Blood pressure was obtained before and $1 \mathrm{~min}$ after the test. Heart rate, $\mathrm{S}_{\mathrm{pO}_{2}}$, perception of dyspnea, and perception of leg fatigue were assessed before, during, and after the 6MWT.

\section{Respiratory Muscle Strength}

Respiratory muscle strength was assessed by measuring maximum inspiratory pressure $\left(\mathrm{P}_{\text {Imax }}\right)$ and maximal expiratory pressure $\left(\mathrm{P}_{\text {Emax }}\right)$ with the MVD-300 $+300 \mathrm{~cm} \mathrm{H}_{2} \mathrm{O}$ digital manovacuometer (Microhard System, Globalmed, Porto Alegre, Brazil). During the procedure, the subjects remained seated at rest, with their trunk erect, nostrils occluded with a nasal clip, and mouth engaged in the mouthpiece to prevent air leakage. For $\mathrm{P}_{\mathrm{Emax}}$ measurement, the subject was asked to inhale to total lung capacity, followed by a maximum expiratory effort. $\mathrm{P}_{\text {Imax }}$ was obtained as the subject inhaled, making use of the total lung capacity and generating a maximum inspiratory effort. Subjects had 3-6 attempts to perform this test, and the evaluator recorded all values obtained for $\mathrm{P}_{\mathrm{Imax}}$ and $\mathrm{P}_{\text {Emax }}$. The highest $\mathrm{P}_{\text {Imax }}$ and $\mathrm{P}_{\text {Emax }}$ values obtained from each subject, which were not $>10 \%$ of the second highest values, were chosen by the evaluator. ${ }^{15}$

\section{1-Min Sit-to-Stand Test}

To perform the 1-min sit-to-stand test, each subject was instructed to sit on a chair with his or her back resting against the back of the chair, and then to stand up fully without utilizing his or her arms, and then to return to a seated position again with his or her back against the back of the chair. Each subject performed as many repetitions as possible in 1 min. ${ }^{16,17}$

\section{Interventions}

Subjects were randomly placed into the step group or the intervention group. The step group performed a physiotherapeutic program standardized by the hospital's team of physical therapists. This intervention started in the immediate postoperative period of cardiac surgery and occurred during the days of hospital stay. The step program (Table 1) consisted of progressive exercises according to each postoperative day. ${ }^{18}$

Two daily sessions with an average duration of $25 \mathrm{~min}$ were performed. Exercise intensity was determined by monitoring the heart rate, which was allowed to reach up to 30 beats/min over the resting heart rate. If the subject presented any signs or symptoms during the evaluated exercise, the evaluation or intervention were interrupted, and the subject was sent to the on-call physician from the coronary unit. These signs or symptoms could be fatigue, chest pain, dyspnea, cyanosis, pallor, tachycardia (> 120 beats/min), bradycardia, complex arrhythmias, or hypotension.

In addition to the physiotherapeutic program standardized by the hospital's team of physical therapists, the intervention group performed physical exercise on a cycle ergometer with CPAP. The step protocol was also started in the immediate postoperative period of cardiac surgery and occurred during the days of hospital stay. Physical exercise on a cycle ergometer combined with CPAP was performed in a single daily session from the second to the fourth postoperative days. From the second postoperative day, subjects started ambulation according to the standardized protocol. For this reason, the dynamic exercise on the cycle ergometer was started. On the fourth postoperative day, the subject carried out the intervention in the morning and was assessed in the afternoon because some patients were discharged on this postoperative day. The exercise time was established according to the subject's vital signs 


\section{Physical Exercise With CPAP}

Table 1. Step Program for the Rehabilitation of Subjects Who Underwent Cardiac Surgery

Step 1: Caloric intake $=2$ MET. Subject lying down (head section raised $\left.45^{\circ}\right)$. Diaphragmatic breathing exercises + ventilatory patterns. Active limb exercises. Manual clearing of the drain. Breathing exercises and flow stimulation + high-frequency oral oscillation (usually 10 repetitions every 2 h). CPAP if necessary.

Step 2: Caloric intake $=2$ MET. Seated subject. Diaphragmatic breathing exercises + ventilatory patterns. Active UL and LL exercises. Manual clearing of the drain. Breathing exercises and flow stimulation + high-frequency oral oscillation. CPAP if necessary.

Step 3: Caloric intake = 3-4 MET. Standing subject. Passive stretching of LL. Active UL and LL exercises. Ambulation: 35-60 m. Breathing exercises and flow stimulation + high-frequency oral oscillation. CPAP if necessary.

Step 4: Caloric intake = 3-4 MET. Standing subject. Passive stretching of LL. Active UL and LL exercises. Active stretching of UL. Ambulation: 60-100 m. Stair training (going down 1 level). Breathing exercises and flow stimulation. CPAP if necessary.

Step 5: Caloric intake = 3-4 MET. Standing subject. Active stretching of UL. Active stretching of LL. Active UL and LL exercises. Ambulation: 100-150 m. Stair training (going up/down 1 level). Breathing exercises and flow stimulation. CPAP if necessary.

Step 6: Caloric intake $=3-4$ MET. Standing subject. Active UL and LL stretching. Active UL and LL exercises. Ambulation: 150-200 m. Stair training (going up/down 2 levels). Breathing exercises and flow stimulation. CPAP if necessary.

Step 7: Caloric intake $=4-6$ MET. Standing subject. Active UL and LL stretching. Active UL and LL exercises. Ambulation: $>200 \mathrm{~m}$. Stair training (going up/down 3 levels). Breathing exercises and flow stimulation. CPAP if necessary.

MET $=$ metabolic equivalents

$\mathrm{UL}=$ upper limbs

$\mathrm{LL}=$ lower limbs

and tolerance. The maximum exercise time for postoperative day 2 was $20 \mathrm{~min}$, and $30 \mathrm{~min}$ for the third and fourth postoperative days. CPAP was delivered (ResMed C-Series Tango) through a face mask (Ultra Mirage, ResMed) with pressure levels of $10 \mathrm{~cm} \mathrm{H}_{2} \mathrm{O}$, based on a previous study. ${ }^{7}$ A single physical exercise session on a cycle ergometer combined with CPAP was performed daily, and the step protocol was performed independently twice a day. Exercise intensity was determined by monitoring the heart rate, which was allowed to reach up to 30 beats/ min when resting; $\mathrm{S}_{\mathrm{pO}_{2}}$ up to $90 \%$ without supplementation with oxygen therapy; and perceived exertion rate between mild and moderate through the Modified Borg Scale. ${ }^{19}$ If a subject presented any signs or symptoms during the evaluated exercise, the same procedure from the step group was adopted.

\section{Statistical Analysis}

SPSS 23.0 (IBM, Armonk, New York) was used for data analysis. The intention-to-treat analysis was not performed because subjects were excluded before the intervention. The Kolmogorov-Smirnov test was applied to evaluate the normality of the data. In the comparisons between pre- and postintervention data, the paired Student $t$ test was used for parametric data, and the Wilcoxon test was used for non-parametric data. The Tukey test was used when a single condition was compared between pre- and postintervention data. The Pearson chi-square test and the Fisher exact test were used for nominal variables. $P \leq .05$ was considered statistically significant.

\section{Results}

From the 59 patients eligible for evaluation, 17 were excluded: 1 for refusing to participate in the study, 8 did not fulfill inclusion criteria ( 2 with orthopedic problems, 3 with angina, 3 who were hemodynamically unstable) and 8 other reasons ( 1 for anticipation of surgery, and 7 with logistical problems). Therefore, a total of 42 individuals were randomized. From these subjects, 11 (5 in the step group, 6 in the experimental group) did not complete the program: not reassessed, 6 for cardiac arrhythmia, 1 for surgical re-intervention, and 2 for death. Therefore, 16 subjects completed the program in the step group, with 15 subjects in the intervention group (Fig. 1).

Thirty-one participants completed the study (mean age $60 \pm 7 \mathrm{y}, 71 \%$ males, body mass index $\left.=29 \pm 5 \mathrm{~kg} / \mathrm{m}^{2}\right)$. Age was significantly lower in the intervention group $(P=$ .048). However, in the regression analysis, this variable did not influence the distance walked on the 6MWT $(P=.72)$. No significant differences were observed in other demographic or clinical characteristics between the groups (Table 2). Length of stay in the ICU was significantly lower in the intervention group. No significant differences were observed in the other variables (Table 3).

The functional capacity measured by the distance walked on the 6MWT in the step group decreased significantly after the intervention from $289.6 \mathrm{~m}$ (range 203.6-375.7) to $180.8 \mathrm{~m}$ (range 120.26-241.4) $(P \leq .001)$. In the intervention group, a reduction in functional capacity was also observed, but it was not significant, dropping from $263.6 \mathrm{~m}$ (range 131.9-395.2) to $216.5 \mathrm{~m}$ (range 141.4-291.5) $(P=.11)$. In both groups, there was a significant decrease 


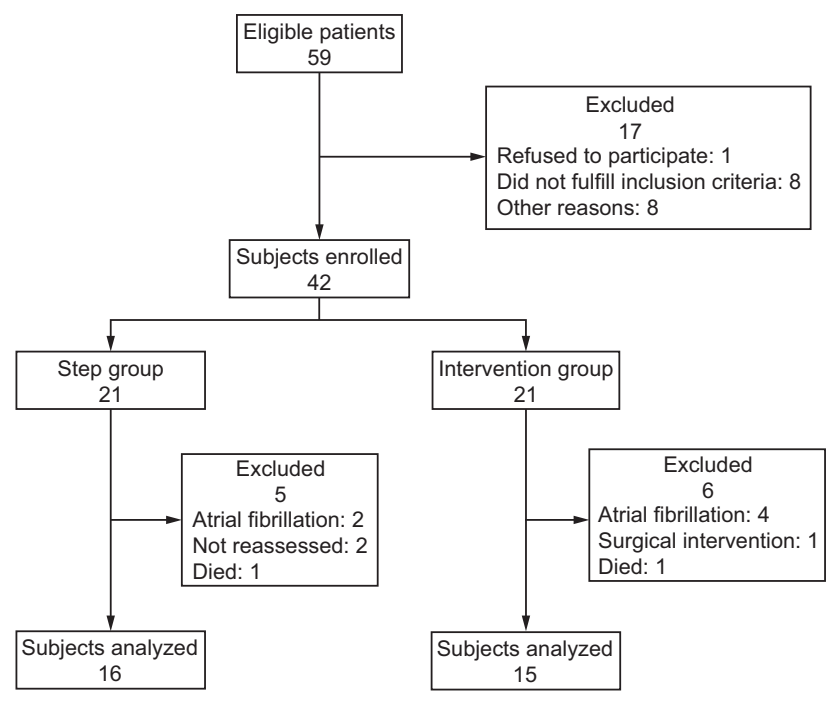

Fig. 1. Flow chart.

Table 2. Sample Characteristics

\begin{tabular}{|c|c|c|c|}
\hline & Step Group & $\begin{array}{l}\text { Intervention } \\
\text { Group }\end{array}$ & $P$ \\
\hline Age, y & $62 \pm 6$ & $57 \pm 8$ & $.048 *$ \\
\hline Males & $10(62.5)$ & $12(80.0)$ & $.43 \dagger$ \\
\hline Weight, kg & $77.39 \pm 14.97$ & $81.33 \pm 21.72$ & $.89^{*}$ \\
\hline Height, $\mathrm{cm}$ & $164.63 \pm 8.56$ & $165.87 \pm 7.49$ & $.75^{*}$ \\
\hline Body mass index, $\mathrm{kg} / \mathrm{m}^{2}$ & $28 \pm 4$ & $29 \pm 6$ & $.87 \ddagger$ \\
\hline Ejection fraction, $\%$ & $62 \pm 11$ & $56 \pm 12$ & $.12 \ddagger$ \\
\hline \multicolumn{4}{|l|}{ Medical history } \\
\hline $\begin{array}{c}\text { Systemic arterial } \\
\text { hypertension }\end{array}$ & $12(75)$ & $15(100)$ & $.10 \dagger$ \\
\hline Diabetes mellitus & $8(50)$ & $9(64)$ & $.43 *$ \\
\hline Acute myocardial infarction & $7(44)$ & $9(60)$ & $.37 *$ \\
\hline Congestive heart failure & $2(12)$ & $5(33)$ & $.22 \dagger$ \\
\hline Dyslipidemia & $10(62)$ & $11(73)$ & $.52 *$ \\
\hline COPD & $3(19)$ & $5(33)$ & $.43 \dagger$ \\
\hline $6 \mathrm{MWD}, \mathrm{m}$ & $289.6 \pm 86.1$ & $263.6 \pm 131.6$ & $.52 *$ \\
\hline $\mathrm{P}_{\text {Imax }}, \mathrm{cm} \mathrm{H}_{2} \mathrm{O}$ & $69 \pm 20$ & $72 \pm 37$ & $.80 \ddagger$ \\
\hline $\mathrm{P}_{\text {Emax }}, \mathrm{cm} \mathrm{H}_{2} \mathrm{O}$ & $86 \pm 34$ & $94 \pm 39$ & $.59 *$ \\
\hline 1-min STS repetitions, no. & $17.4 \pm 5$ & $18.5 \pm 5$ & $.53 *$ \\
\hline $\begin{array}{l}\text { Data are presented as mean } \pm \mathrm{SD} \text { or } n \text { (\%) } \\
* \text { Student } t \text { test. } \\
\dagger \text { Fischer exact test. } \\
\text { ‡ Wilcoxon test. } \\
\text { 6MWD = 6-min walk distance } \\
\mathrm{P}_{\text {Imax }}=\text { maximum inspiratory pressure } \\
\mathrm{P}_{\text {Emax }} \text { maximum expiratory pressure } \\
1 \text {-min } \mathrm{STS}=1 \text {-min sit-to-stand test }\end{array}$ & t). $P \leq .05$ indicates st & tatistical significance. & \\
\hline
\end{tabular}

in $\mathrm{P}_{\text {Imax }}, \mathrm{P}_{\mathrm{Emax}}$, and 1-min sit-to-stand test on the postoperative day 4 compared to the preoperative period (Table 4).

Figure 2 shows the distances walked on the 6MWT in the step group and in the intervention group in the preoperative and postoperative periods. The best performance in
Table 3. Perioperative Data From Subjects

\begin{tabular}{lccl}
\hline \hline & Step Group & Intervention Group & $P$ \\
\hline Surgical duration, min & $189.3 \pm 25.1$ & $189.7 \pm 33.1$ & $.82 *$ \\
Number of bypasses & $3.00 \pm 0.73$ & $2.87 \pm 0.92$ & .73 \\
Duration of ventilation, min & $637.5 \pm 244.9$ & $579.0 \pm 105.4$ & .69 \\
ICU length of stay, d & $2.9 \pm 0.7$ & $2.5 \pm 0.5$ & .050 \\
Hospital length of stay, d & $7.3 \pm 1.2$ & $7.1 \pm 1.1$ & .69
\end{tabular}

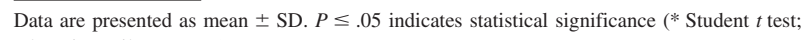
otherwise Wilcoxon test).

Table 4. Pre- and Postintervention Comparison Between the Step Group and the Intervention Group

\begin{tabular}{|c|c|c|c|}
\hline & Step Group & Intervention Group & $P$ \\
\hline \multicolumn{4}{|l|}{$6 \mathrm{MWD}, \mathrm{m}$} \\
\hline Pre & $289.63 \pm 86.07$ & $263.60 \pm 131.65$ & .52 \\
\hline Post & $180.81 \pm 60.55$ & $216.47 \pm 75.07$ & .16 \\
\hline Mean $\neq$ intra & $109(38 \%)$ & $47(18 \%)$ & \\
\hline$P$ & $\leq .001$ & .11 & \\
\hline \multicolumn{4}{|l|}{$\mathrm{P}_{\text {Imax }}, \mathrm{cm} \mathrm{H}_{2} \mathrm{O}$} \\
\hline Pre & $69.00 \pm 20.38$ & $71.73 \pm 36.79$ & .80 \\
\hline Post & $46.81 \pm 23.53$ & $47.33 \pm 19.80$ & .95 \\
\hline Mean $\neq$ intra & $23(34 \%)$ & $24(35 \%)$ & \\
\hline$P$ & .001 & .003 & \\
\hline \multicolumn{4}{|l|}{$\mathrm{P}_{\mathrm{Emax}}, \mathrm{cm} \mathrm{H}_{2} \mathrm{O}$} \\
\hline Pre & $86.38 \pm 34.14$ & $93.64 \pm 38.60$ & .59 \\
\hline Post & $56.06 \pm 20.19$ & $67.00 \pm 19.15$ & .13 \\
\hline Mean $\neq$ intra & $30(35 \%)$ & $26(28 \%)$ & \\
\hline$P$ & .001 & .002 & \\
\hline \multicolumn{4}{|c|}{ 1-min STS repetitions, no. } \\
\hline Pre & $17.38 \pm 4.91$ & $18.50 \pm 4.67$ & .53 \\
\hline Post & $10.83 \pm 3.59$ & $13.36 \pm 4.72$ & .16 \\
\hline Mean $\neq$ intra & $7(42 \%)$ & $5(28 \%)$ & \\
\hline$P$ & .003 & .032 & \\
\hline \multicolumn{4}{|c|}{$\begin{array}{l}\text { Data are presented as mean } \pm \text { SD. } P \leq .05 \text { indicates statistical significance. Step group: } \\
n=16 \text {; Intervention group: } n=15 \text {. }\end{array}$} \\
\hline $\begin{array}{l}\text { Mean } \neq \text { intra }=\text { mean } \\
\text { ANOVA }(\text { for intergro } \\
6 \mathrm{MWD}=6 \text {-min walk } \\
\mathrm{P}_{\text {Imax }}=\text { maximum ins } \\
\mathrm{P}_{\text {Emax }}=\text { maximum ex } \\
1 \text {-min STS }=1 \text {-min }\end{array}$ & ference using Wilcoxon & test (for intragroup) and one & \\
\hline
\end{tabular}

the test occurred in the intervention group after the surgical procedure.

\section{Discussion}

This study aimed to evaluate the effects of 2 physical therapy interventions on the rehabilitation of cardiac surgery subjects during the postoperative period. One intervention was a protocol of standardized and progressive exercises known as step, which consisted of an individualized program of physiotherapeutic intervention during 

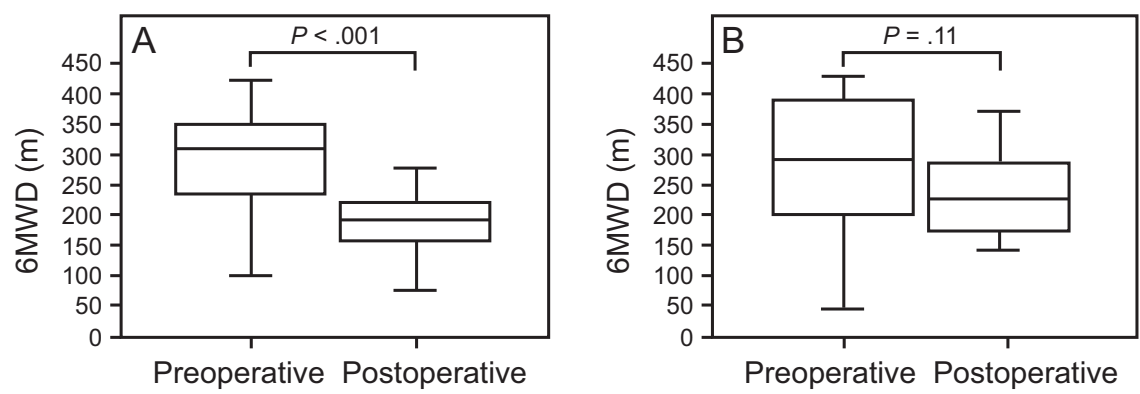

Fig. 2. Functional capacity in (A) the step group and in (B) the intervention group evaluated with the 6-min walk test (Wilcoxon test) in the pre- and postoperative periods. $6 \mathrm{MWD}=6$-min walk distance.

hospital convalescence for patients who underwent CABG. We compared this standardized protocol with a new physiotherapeutic intervention that combines physical exercise on a cycle ergometer with CPAP. The findings were shorter ICU length of stay and maintenance of functional capacity in subjects who performed physical exercise with CPAP compared to those who only performed the step protocol. There was no additional beneficial effect on respiratory muscle strength and lower limb muscle resistance.

In this study, ICU length of stay was shorter in the group that performed physical exercise with CPAP. To our knowledge, our study is the first to demonstrate that length of ICU stay decreased with the addition of physical exercise on a cycle ergometer with CPAP. This important outcome suggests a significant impact on the final cost due to this reduction in the ICU length of stay. A similar result was demonstrated in another study, ${ }^{20}$ which used respiratory muscle training as the physiotherapeutic approach. However, this result was not observed in subjects who performed only $5 \mathrm{~min}$ of physical training on a cycle ergometer in the Fowler position during the ICU stay. ${ }^{10}$ In the analysis of hospital length of stay, we observed no differences between the groups. However, the mean length of hospital stay observed in our results was lower than those described in the literature. ${ }^{10,21,22}$ This result reinforces the importance of the physiotherapeutic intervention independently from the protocol adopted in the present study.

Previous studies have shown that surgical intervention decreases the distance walked on the $6 \mathrm{MWT}^{11,23}$ as well as the respiratory muscle strength ${ }^{24,20}$ when comparing the preoperative period with the postoperative period (evaluated before hospital discharge). In this context, the hospital physiotherapeutic intervention seeks to diminish this reduction, allowing the patient to obtain better physical and functional conditions at hospital discharge, as well as reducing the ICU length of stay and the overall hospital length of stay. ${ }^{20,21,22}$ Our results indicate that physical exercise on a cycle ergometer combined with CPAP is effective because it reduced the loss of functional capability. Another study also reported the beneficial effects of iso- lated physical training, but the intervention occurred over a longer period. ${ }^{10}$ Thus, this suggests that intervention combined with physical exercise on a cycle ergometer with CPAP brings additional beneficial effects. However, studies that examine the comparative effects between isolated physical training on a cycle ergometer and this training associated with CPAP are still needed. Other studies $^{11,23}$ did not show additional effects of isolated physical training using a stationary bicycle when compared to ambulation. In our study, functional capacity was recovered in subjects who performed the physical exercise combined with CPAP, demonstrating a benefit when compared with the step group.

Regarding inspiratory and expiratory muscle strength, the intervention group exhibited no additional beneficial effect. Pre and postoperative analyses showed that respiratory muscle strength was reduced in both groups. Physical therapy treatment seeks to minimize this reduction with different sorts of approaches. Our results corroborate previous studies ${ }^{20,24}$ that did not report any effects on respiratory muscle strength. These studies carried out more specific interventions through inspiratory muscle training. It is known, however, that CABG procedures result in alterations in ventilatory mechanics, and that these alterations often take months to fully recover after hospital discharge. ${ }^{25}$ Thus, although a significant reduction in muscle strength persists until hospital discharge, given the mechanical disadvantage caused by surgery, this disadvantage could have been reduced with the association of exercises and CPAP, a fact supported by the maintenance of functional capacity.

Similar to the results for respiratory muscle strength, lower limb muscle resistance showed a significant reduction from the preoperative to the postoperative periods. The group that performed exercise on a cycle ergometer combined with CPAP improved their performance. This may have contributed to the improvement observed in the distance walked on the 6MWT. Corroborating our findings, other authors observed that a progressive CPAP training program demonstrated beneficial effects on exercise tolerance. ${ }^{7}$ Another study, which used a different therapy 


\section{Physical Exercise With CPAP}

(ie, respiratory muscle training), also obtained positive results in maintaining functional capacity in cardiac surgery subjects during the postoperative period. ${ }^{26}$ These findings, taken together, may indicate that ventilatory muscle relief, or even ventilatory muscle training, which aims to increase the strength of these muscles, may minimize the effects of intolerance to physical exercise in cardiac surgery patients during the postoperative period, ${ }^{27}$ given that this procedure is closely associated with important losses in pulmonary function. ${ }^{28}$

Although the 2 protocols presented in this study showed benefits to subjects, the intervention group protocol delivered additional improvements, mainly in the primary outcome variable. This shows that the interventions during the physical therapy sessions have clinical applicability and important functional outcomes that decrease the length of hospital stay, thus resulting in a reduction of the total hospital cost, even for short hospital stays. In our study, independently from the protocol used, no intercurrence was observed during the rehabilitation sessions. This indicates that the interventions ensure patient recovery and safety in the performance of physical therapy.

\section{Study Limitations}

Our study had two main limitations. First, we were unable to blind the subject and the physical therapist to the type of treatment. Second, we were unable to evaluate the subject in the immediate postoperative period to compare the outcome variables between the pre- and postintervention periods.

\section{Conclusions}

Physical exercise on a cycle ergometer combined with CPAP as an adjunct to the rehabilitation of hospitalized subjects who underwent myocardial revascularization with CABG was safe, decreased the length of stay in the ICU, and helped maintain functional capacity. The results showed an insignificant reduction in the distance walked in the 6MWT in the intervention group. This is very important data and can be explored in further research, given that the utilization of CPAP in association with exercise is poorly explored in these patients. This physical exercise did not have an effect on respiratory muscle strength and peripheral muscle resistance compared to progressive interventional protocols used in hospital physical therapy, also known as the step protocol.

\section{REFERENCES}

1. Bienert IRC, Rodrigues Harada EA, Silva KL, Valente AR, da Silva PA, et al. Avaliação temporal dos procedimentos de revascularização coronariana pelo sistema único de saúde (SUS) no Brasil: um panorama de 20 Anos. Int J Cardiovasc Sci 2017;30(5):380-390.
2. Souza e Silva CG de, Klein CH, Godoy PH, Souza e Silva NA de. Tendências e letalidade hospitalar dos procedimentos de revascularização miocárdica pagos pelo SUS no estado do Rio de Janeiro, 1999-2010. Int J Cardiovasc Sci 2016;29(6):477-491.

3. McWilliams D, Weblin J, Atkins G, Bion J, Williams J, Elliott C, et al. Enhancing rehabilitation of mechanically ventilated patients in the intensive care unit: a quality improvement project. J Crit Care 2015;30(1):13-18.

4. Fanari Z, Barekatain A, Kerzner R, Hammami S, Weintraub WS, Maheshwari V. Impact of a multidisciplinary team approach including an intensivist on the outcomes of critically ill patients in the cardiac care unit. Mayo Clin Proc 2016;91(12):1727-1734.

5. Mehaffey JH, Hawkins RB, Byler M, Charles EJ, Fonner C, Kron I, et al. Cost of individual complications following coronary artery bypass grafting. J Thorac Cardiovasc Surg 2018;155(3):875882 .

6. Herdy AH, López-Jiménez F, Terzic CP, Milani M, Stein R, Carvalho $\mathrm{T}$, et al. Sociedade Brasileira de Cardiologia. Diretriz SulAmericana de prevenção e reabilitação cardiovascular. Arq Bras Cardiol 2014;103(2 Suppl 1):1-31.

7. Pantoni CBF, Di Thommazo-Luporini L, Mendes RG, Caruso FCR, Mezzalira D, Arena R, et al. Continuous positive airway pressure during exercise improves walking time in patients undergoing inpatient cardiac rehabilitation after coronary artery bypass graft surgery: a randomized controlled trial. J Cardiopulm Rehabil Prev 2016;36: 20-27.

8. Borghi-Silva A, Trimer R, Mendes RG, Arena RA, Schwartzmann PV. Rehabilitation practice patterns for patients with heart failure: the South American perspective. Heart Fail Clin 2015;11(1):73-82.

9. Borghi-Silva A, Mendes RG, Trimer R, Cipriano G Jr. Current trends in reducing cardiovascular disease risk factors from around the world: focus on cardiac rehabilitation in Brazil. Prog Cardiovasc Dis 2014; 56(5):536-542.

10. Borges DL, Silva MG, Silva LN, Fortes JV, Costa ET, Assunção RP, et al. Effects of aerobic exercise applied early after coronary artery bypass grafting on pulmonary function, respiratory muscle strength, and functional capacity: a randomized controlled trial. J Phys Act Health 2016;13:946-951.

11. Trevisan MD, Lopes DG, Mello RG, Macagnan FE, Kessler A. Alternative physical therapy protocol using a cycle ergometer during hospital rehabilitation of coronary artery bypass grafting: a clinical trial. Braz J Cardiovasc Surg 2015;30(6):615-619.

12. Zhu G, Huang Y, Wei D, Shi Y. Efficacy and safety of noninvasive ventilation in patients after cardiothoracic surgery: a PRISMA-compliant systematic review and meta-analysis. Medicine (Baltimore) 2016;95(38):e4734

13. World Health Organization. Physical status: the use and interpretation of anthropometry. Report of a World Health Organization Expert Committee. WHO. Technical Report Series No. 854 1995;1452.

14. ATS statement: guideline for the six-minute walk test. Am J Respir Crit Care Med 2002;166(1):111-117.

15. Neder JA, Andreoni S, Lerario MC, Nery LE. Reference values for lung function tests. II. Maximal respiratory pressures and voluntary ventilation. Braz J Med Biol Res 1999;32(6):719-727.

16. Araújo CGS. Teste de sentar-levantar - apresentação preliminar de um procedimento para avaliação em Medicina do Exercício e do Esporte. Rev Bras Med Esporte 1999;5(5):179-182.

17. Gruet M, Peyré-Tartaruga LA, Mely L, Vallier JM. The 1-minute sit-to-stand test in adults with cystic fibrosis: correlations with cardiopulmonary exercise test, 6-minute walk test, and quadriceps strength. Respir Care 2016;61(12):1620-1628.

18. Winkelmann ER, Dallazen F, Bronzatti ABS, Lorenzoni JCW, Windmöller P. Analysis of steps adapted protocol in cardiac re- 


\section{Physical Exercise With CPAP}

habilitation in the hospital phase. Braz J Cardiovasc Surg 2015; 30(1):40-48.

19. Borg GA. Psychophysical bases of perceived exertion. Med Sci Sports Exerc 1982;14(5):377-381.

20. Matheus GB, Dragosavac D, Trevisan P, Costa CE da, Lopes MM, Ribeiro GCdeA. Treinamento muscular melhora o volume corrente e a capacidade vital no pós-operatório de revascularização do miocárdio. Rev Bras Cir Cardiovasc 2012;27(3):362-69.

21. Tashiro N, Takahashi S, Takasaki T, Katayama K, Taguchi T, Watanabe M, et al. Efficacy of cardiopulmonary rehabilitation with adaptive servo-ventilation in patients undergoing off-pump coronary artery bypass grafting. Circulation 2015;79:1290-1298.

22. Cacau LdeA, Oliveira GU, Maynard LG, Araújo Filho AA, Silva WM Jr, Cerqueira Neto ML, et al. The use of the virtual reality as intervention tool in the postoperative of cardiac surgery. Rev Bras Cir Cardiovasc 2013;28(2):281-289.

23. Hirschhorn AD, Richards DA, Mungovan SF, Morris NF, Adams L. Does the mode of exercise influence recovery of functional capacity in the early postoperative period after coronary artery bypass graft surgery? A randomized controlled trial. Interact Cardiovasc Thorac Surg 2012;15:995-1003.
24. Cordeiro ALL, Melo TA de, Neves D, Luna J, Esquivel MS, Guimarães ARF, et al. Inspiratory muscle training and functional capacity in patients undergoing cardiac surgery. Braz J Cardiovasc Surg 2016;31(2):140-144.

25. Borghi-Silva A, Mendes RG, Costa F de SM, Di Lorenzo VAP, Oliveira CR de, Luzzi S. The influences of positive end expiratory pressure (PEEP) associated with physiotherapy intervention in phase I cardiac rehabilitation. Clinics 2005;60(6):465-472.

26. Ximenes NNPS, Borges DL, Lima RO, Barbosa e Silva MG, Silva LN, Costa Mde A, et al. Effects of resistance exercise applied early after coronary artery bypass grafting: a randomized controlled trial. Braz J Cardiovasc Surg 2015;30(6):620-625.

27. Pantoni CB, Di Thommazo L, Mendes RG, Catai AM, Luzzi S, Amaral Neto O, Borghi-Silva A. Effects of different levels of positive airway pressure on breathing pattern and heart rate variability after coronary artery bypass grafting surgery. Braz J Med Biol Res 2011;44(1):38-45.

28. Caruso FC, Simões RP, Reis MS, Guizilini S, Alves VL dos S, Papa $\mathrm{V}$, et al. High-intensity inspiratory protocol increases heart rate variability in myocardial revascularization patients. Braz J Cardiovasc Surg 2016;31(1):38-44.

This article is approved for Continuing Respiratory Care Education credit. For information and to obtain your CRCE

(free to AARC members) visit www.rcjournal.com

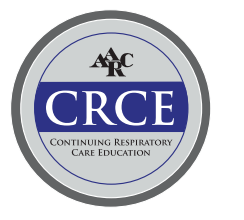

